\title{
VI Deregulierung statt Liberalisierung: Keine Regelung der Namensbestimmung als Alternative?
}

Abschließend sei die ketzerische Frage gestattet, ob sich der Staat nicht vollkommen aus der Regulierung der Namensbestimmung zurückziehen sollte, wie das in der Geschichte des Personenrechts überwiegend der Fall war. Der Personenname war auch bei uns lange Zeit ein außerrechtliches Phänomen, geprägt vor allem von Sitte und Brauchtum. ${ }^{166}$ Noch im Band 45 des Archivs für die civilistische Praxis schreibt im Jahr 1862 ein Privatdozent aus Jena unter der Abschnittsüberschrift „Oeffentlich-rechtliches Interesse am Namenswesen“: „Die allgemeine Ansicht der das Namensrecht berührenden Schriftsteller geht, mit einer Ausnahme [...], dahin: der Name und zwar Vor- wie Geschlechtsname sei durch und durch Privatsache; der Staat habe nicht das geringste Interesse am Namenswesen an sich; er überlasse daher Jedem Auswahl, Gebrauch oder Nichtgebrauch seines Namens, gebe dem Einzelnen volle Willkühr, den irgendwie erlangten Namen zu führen, denselben abzuändern oder mit irgend einem andern Namen zu vertauschen“. 167

Bei näherem Hinsehen ist die Frage nach dem Ob einer Regelung des Personennamens durchaus berechtigt, da das staatliche Regulierungspotential im Namensrecht nicht besonders groß ist. Zunächst zwingt das Recht - auch wenn es den Namen bestimmt - den Namensträger grundsätzlich nicht, in der Gesellschaft den rechtlichen Namen zu führen. Anders als bei der Firma, die der Kaufmann führen muss (oben V. 1.), steht es jedem Bürger frei, Pseudonyme zu verwenden $^{168}$ - ein Recht, von dem viele vor allem bei Aktivitäten im Internet Gebrauch machen, aber auch im gesellschaftlichen Verkehr, wenn sie Ruf-, Spitz-, Kurz- oder Künstlernamen verwenden. Dies gilt sogar für die Namensführung in der Ehe. Die meisten romanischen Rechtsordnungen, die keinen gemeinsamen Ehenamen kennen, geben den Ehegatten das Recht, den Namen des anderen Ehegatten neben ihrem rechtlichen Namen zu gebrauchen. ${ }^{169}$ Aber selbst bei uns müssen Ehegatten einen von ihnen rechtlich bestimmten Ehenamen entgegen der

166 Vom „langen Weg des Namens zum Recht“ spricht etwa Schwab (Fn. 36).

167 Robert Hermann Über das Recht der Namensführung und der Namensänderung, AcP 45 (1862) 153 (160 f.), der allerdings in seinem Beitrag die Gegenthese zu begründen versucht.

168 So bereits Opet (Fn. 40) $324 \mathrm{ff}$.

169 Näher etwa Walter Pintens Die Anwendung des belgischen, französischen und niederländischen Namensrechtsrechts durch den deutschen Standesbeamten, StAZ 1984, 188 (189 f.); ders. Der Personenname in der romanischen Rechtsfamilie, StAZ 2016, 65 (71 f.). 
missverständlichen Formulierung in $§ 1355$ Abs. 1 S. 2 BGB („Die Ehegatten führen den von ihnen bestimmten Ehenamen“) nicht mit der Folge führen, dass bei Nichtverwendung des Ehenamens der eine Ehegatte den anderen auf Herstellung der ehelichen Lebensgemeinschaft in Anspruch nehmen kann. ${ }^{170}$ Auch die Rechtsgeschäftslehre des bürgerlichen Rechts vermag ein „Handeln unter falschem Namen“ adäquat zu bewältigen. Es ist anerkannt, dass beispielsweise beim Vertragsschluss die handelnde Person Vertragspartei wird, solange die Verwendung des - rechtlich - falschen Namens ,bei der anderen Vertragspartei keine Fehlvorstellung über die Identität des Handelnden hervor[...]ruf[t] [...], diese den Vertrag also nur mit dem Handelnden schließen will“. ${ }^{171}$ Es wird mithin niemand gezwungen, seinen rechtlichen Namen in der Gesellschaft zu führen.

In welchen Situationen aber ist der rechtliche Name ausnahmsweise $\mathrm{zu}$ führen? Denn schließlich erfasst der Staat die rechtlichen Namen seiner Bürger personenstandsrechtlich, und zwar weitgehend lückenlos, selbst bei Fällen mit Auslandsbezug, vgl. $\S 34$ ff. PStG: Der rechtliche Name ist Teil des Personenstands, wie ausdrücklich $\S 1$ Abs. 1 S. 1 PStG klarstellt, wonach der Personenstand „die sich aus den Merkmalen des Familienrechts ergebende Stellung einer Person innerhalb der Rechtsordnung einschließlich ihres Namens“ umfasst. Auch die Meldebehörden speichern - nach Mitteilung durch die Standesämter gemäß § 17 Abs. 4 des Bundesmeldegesetzes ${ }^{172}$ (BMG) - den rechtlichen Namen der Bürger, einschließlich früherer Namen, im Melderegister (§3 Abs. 1 Nr. 1-3 BMG), um diese Daten anderen öffentlichen Stellen zur Verfügung zu stellen. Der rechtliche Name wird daher auch von den Behörden verwendet, wenn sie amtliche Dokumente - allen voran Ausweisdokumente - ausstellen. Im Verkehr mit Behörden und Amtsträgern ist stets der rechtliche Name zu verwenden, wie in erster Linie das Ordnungswidrigkeitenrecht sicherstellt: Nach $\S 111$ Abs. 1 des Gesetzes über Ordnungswidrigkeiten ${ }^{173}$ (OWiG) handelt der Namensträger ordnungswidrig, wenn dieser „einer zuständigen Behörde, einem zuständigen Amtsträger oder einem zuständigen Soldaten der Bundeswehr über seinen Vor-, Familien- oder Geburtsnamen [...] eine unrichtige Angabe macht oder die Angabe verweigert". Es besteht sogar eine fahrlässige Ordnungswidrigkeit, vgl. §111 Abs. 2 OWiG. Auch

170 So etwa bereits zutreffend Bernhard Raschauer Namensrecht (1978) S. 249; für ein wechselseitiges und gerichtlich im Wege des positiven Herstellungsantrags durchsetzbares Recht der Ehegatten, vom jeweils anderen Ehegatten die Führung des Ehenamens zu verlangen, aber etwa Brudermüller in: Palandt, Bürgerliches Gesetzbuch, 79. Aufl. 2020, vor § 1353 BGB Rn. 13.

171 Siehe etwa BGH 8.12.2005, NJW-RR 2006, 701 (702).

172 Vom 3.5.2013, BGBl. 2013 I S. 1084.

173 In der Fassung der Bekanntmachung vom 19.2.1987, BGBl. 1987 I S. 602. 
Amtsträger sind verpflichtet, ihren rechtlichen Namen zu führen. ${ }^{174}$ Rechtlich nicht über alle Zweifeln erhaben war deshalb die Praxis des ehemaligen Bundespräsident Richard Karl Freiherr von Weizsäcker, von ihm nach Art. 82 Abs. 1 S. 1 GG ausgefertigte Gesetze mit dem verkürzten Familiennamen „Weizsäcker“ zu unterzeichnen. ${ }^{175}$ Auch im Privatrechtsverkehr verpflichtet das Recht die Bürger ausnahmsweise zur Verwendung ihres rechtlichen Namens, den sie mit amtlichen Ausweisdokumenten nachweisen müssen. Das Geldwäschegesetz ${ }^{176}$ (GwG) z. B. verpflichtet in $\S 11$ Abs. 4 Nr. 1 lit. a GwG neben anderen Personen und Institutionen Kredit- und Finanzinstitute, aber auch Rechtsanwälte und Notare bei bestimmten Transaktionen vor Begründung der Geschäftsbeziehung oder Durchführung der Transaktion (so der Grundsatz nach $\S 11$ Abs. 1 S. 1 GwG) den rechtlichen Namen eines Vertragspartners - das Gesetz spricht vom Vor- und Nachnamen - zu erheben, soweit es sich beim Vertragspartner um eine natürliche Person handelt. Mit diesen Pflichten zur Namensführung möchte der Staat die Individualisierungsfunktion des Namens (oben III. 1.) nutzen. Da der rechtliche Name als Individualisierungsmittel aber, wie gesehen (oben III. 1.), nur bedingt tauglich ist, kann der Staat auch mit Namensführungspflichten nur eingeschränkt eine Individualisierung sicherstellen. Eine Individualisierung seiner Bürger würde der Staat daher allein über die Pflicht zur Führung einer einmalig vergebenen Identifikationsnummer sicherstellen.

Trotz dieses fehlenden Regulierungspotentials und eingeschränkten Regulierungswecks kann sich das Recht aus dem Namenswesen nicht zurückziehen, jedenfalls nicht im Alleingang. Solange andere Staaten den Namen als Individualisierungsmittel nutzen, muss der Staat jedenfalls im grenzüberschreitenden Personenverkehr Ausweisdokumente mit Namen ausstellen. Innerhalb der Europäischen Union muss jeder Mitgliedstaat etwa in seinen Reisepässen für seine Bürger den Namen angeben. Diese Vorgabe folgt aus den Standards der International Civil Aviation Organisation (ICAO), welche die Europäische Union für die

174 Für Notare etwa OLG Köln 1.2.1977, FamRZ 1978, 680 (681).

175 Worauf - freilich mit etwas zu viel Emphase (vgl. auch die Rezension von Wacke FamRZ 1999, 977 und von Hepting StAZ 1998, 354) - Sebastian-Johannes von Spoenla-Metternich Namenserwerb, Namensführung und Namensänderung unter Berücksichtigung von Namensbestandteilen (1997) S. 130, hingewiesen hat, wonach der damalige Bundespräsident auf diese Weise suggeriert habe, dass die ehemalige Adelsbezeichnung „Freiherr von“ kein rechtlicher Namensbestandteil sei, sondern ein Titel, den man führen kann, aber nicht führen muss, anders als den Namen im amtlichen Verkehr.

176 Gesetz über das Aufspüren von Gewinnen aus schweren Straftaten vom 23.6.2017, BGBl. 2017 I S 1822. 
Gestaltung der mitgliedstaatlichen Reisepässe kraft einer Verordnung ${ }^{177}$ verbindlich gemacht hat. ${ }^{178}$ Sobald der Staat allerdings den Namen als ein künstliches Merkmal des jeweiligen Bürgers erfasst, kann es sich nur um einen rechtlichen - weil jedenfalls für diese Erfassung verbindlichen - Namen handeln und es bedarf Regeln zur Namensbestimmung (und sei es auch nur die Regel, dass der Bürger seinen Namen frei wählen kann).

Jedoch kann die Tatsache, dass keine allgemeine Namensführungspflicht besteht, nicht gegen eine Liberalisierung des Namensrechts (oben V.) ins Feld geführt werden. ${ }^{179}$ Zwar haben das Bundesverfassungsgericht und der Straßburger Gerichtshof in der Vergangenheit bei der grund- und menschenrechtlichen Prüfung namensrechtlicher Regelungen auf das eben geschilderte geringe Regelungspotential hingewiesen, die Einschränkungen der Namenswahlfreiheit weniger drastisch erscheinen lassen. ${ }^{180}$ Allerdings hat sich auch in der verfas-

177 Verordnung (EG) Nr. 2252/2004 des Rates vom 13.12.2004 über Normen für Sicherheitsmerkmale und biometrische Daten in von den Mitgliedstaaten ausgestellten Pässen und Reisedokumenten, ABl. 2004, L 385/1.

178 EuGH 2.10.2014, Rs. C-101/13 (U gegen Stadt Karlsruhe) StAZ 2015, 104, Rn. 26, sowie Rn. 28 ff. (zum Namensbegriff).

179 Vgl. aber Vorschläge, neben dem rechtlichen Geburtsnamen, der unveränderlich bleibt, die spätere Namensführung über Gebrauchsnamen der Sitte anheimzustellen, von Coester (Fn. 43) 303 f.; ders. Das neue Familienrechtsnamensgesetz, FuR 1994, 1 (8); Schwenzer (Fn. 43) 395; Hepting (Fn. 16) 121; Gaaz (Fn. 18) 165.

180 So etwa BVerfG 8.3.1988, BVerfGE 78, 38 = StAZ 1988, 164 = FamRZ 1988, 587 (589): „Damit wird dem allgemeinen Persönlichkeitsrecht des Ehegatten, dessen Name weicht, hinreichend Rechnung getragen. Formal betrachtet, büßt der ,weichende‘ Ehegatte zwar seinen Namen ein. Den bisherigen Namen darf er - rechtlich gesehen - nur voranstellen. In den praktischen Auswirkungen kommt das aber einer Beifügung des Ehenamens gleich. Im täglichen Umgang kann er den bisher geführten Namen beibehalten, unter dem er sich einen ,Namen gemacht` hat. Die Funktion des Namens, die Individualität seines Trägers auszudrücken, wird daher im wesentlichen gewahrt“; EGMR 6.5.2008, Nr. 33572/02 (Alexandra von Rehlingen and others against Germany), deutsche Übersetzung abgedruckt in StAZ 2008, 375: „Having regard to the specific circumstances of the case, the Court notes that the applicant children had been able to use their compound names within their social sphere and would continue to do so. The fact that the use of their compound names in everyday life did not create any practical problems does however not, in the Court's view, contradict the legislator's general decision to restrict the official passing on of compound names to children in order to avoid disadvantages for future generations"; BVerfG 5.5. 2009, BVerfGE 123, 90 = StAZ 2009, 179 = FamRZ 2009, 939 (942): „Selbst wenn aber die Ehegatten den Doppelnamen eines Ehegatten zum Ehenamen bestimmen, sodass der bisher vom anderen Ehegatten geführte Name gemäß § 1355 Abs. 4 S. 2 BGB zum Wegfall kommt und selbst als Begleitname nicht mehr rechtliche Anerkennung findet, bleibt es dem betroffenen Ehegatten weiterhin unbenommen, im Geschäftsverkehr mit seinem bisher geführten Namen zu firmieren (§ 21 HGB) und den Namen zusammen mit seinem Ehenamen zu tragen. Das deutsche Namensrecht schreibt 
sungsrechtlichen Diskussion die Bedeutung des Personenstands - so wie er vom Staat erfasst wird - gewandelt. Erst kürzlich hat das Bundesverfassungsgericht die Argumentation, dass das Geschlecht des Menschen zwar personenstandsrechtlich erfasst wird, aber dieser Eintrag wenig soziale Relevanz besitzt, weil der Einzelne in der Gesellschaft frei ist, sich einem Geschlecht zuzuordnen, ${ }^{181}$ nicht gelten lassen. Vielmehr sei der Gesetzgeber verfassungsrechtlich verpflichtet, personenstandsrechtlich einen positiven Geschlechtseintrag für Personen vorzusehen, die sich weder dem männlichen noch dem weiblichen Geschlecht zuordnen lassen: „Unter den gegebenen Umständen hat die personenstandsrechtliche Anerkennung [...] Identität stiftende und ausdrückende Wirkung. Der Personenstand ist keine Marginalie, sondern ist nach dem Gesetz die ,Stellung einer Person innerhalb der Rechtsordnung، (§1 Abs. 1 S. 1 PStG). Mit dem Personenstand wird eine Person nach den gesetzlich vorgesehenen Kriterien vermessen; er umschreibt in zentralen Punkten die rechtlich relevante Identität einer Person. Daher gefährdet die Verwehrung der personenstandsrechtlichen Anerkennung [...] bereits an sich, das heißt unabhängig davon, welche Folgen außerhalb des Personenstandsrechts an den [...][E]intrag geknüpft sind, die selbstbestimmte Entwicklung und Wahrung der Persönlichkeit einer Person spezifisch“. ${ }^{182}$ Das Personenstandsrecht ist offenbar mehr als reines Registerrecht. Der staatlich erfasste Personenstand wird zunehmend materialisiert. Diese Materialisierung bedeutet für die Ausgestaltung des Namensrechts, dass beim in den Personenstandsregistern eingetragenen Namen keine Abstriche gemacht werden dürfen - verfassungsrechtlich nicht, aber auch rechtspolitisch nicht, konkret nicht von der Verwirklichung der Selbstdarstellungsfunktion durch eine grundsätzlich umfassende Namenswahlfreiheit.

keine starre Namensführung vor und lässt es ausreichen, wenn mit der Namensunterschrift die eindeutige Identifizierung der Person möglich ist. Lediglich gegenüber Behörden ist der rechtlich anerkannte Name, in diesem Fall der Ehedoppelname, anzugeben [...]. Dies stellt nur eine geringe Beeinträchtigung des Persönlichkeitsrechts dar [...]“.

181 So etwa von Dutta/Helms Geschlechtseintrag ,inter/divers“ im Geburtenregister? StAZ 2017, 98 (101 ff.).

182 BVerfG 10.10.2017, BVerfGE 147, 1 = StAZ 2018, 15 = FamRZ 2017, 2046, Rn. 45. 